P0063

\title{
SPATIALLY RESOLVED MEASUREMENTS OF DIFFUSE REFLECTANCE
}

\author{
Nina Basic et al.
}

DOI 10.25039/x46.2019.PO063

from

CIE x046:2019

Proceedings

of the

29th CIE SESSION

Washington D.C., USA, June 14 - 22, 2019

(DOI 10.25039/x46.2019)

The paper has been presented at the 29th CIE Session, Washington D.C., USA, June 14-22, 2019. It has not been peer-reviewed by CIE.

(C) CIE 2019

All rights reserved. Unless otherwise specified, no part of this publication may be reproduced or utilized in any form or by any means, electronic or mechanical, including photocopying and microfilm, without permission in writing from CIE Central Bureau at the address below. Any mention of organizations or products does not imply endorsement by the CIE.

This paper is made available open access for individual use. However, in all other cases all rights are reserved unless explicit permission is sought from and given by the CIE.

CIE Central Bureau

Babenbergerstrasse 9

A-1010 Vienna

Austria

Tel.: +4317143187

e-mail: ciecb@cie.co.at

www.cie.co.at 


\title{
SPATIALLY RESOLVED MEASUREMENTS OF DIFFUSE REFLECTANCE
}

\author{
Basic, N. ${ }^{1}$, Blattner, P. ${ }^{1}$, Pastuschek, M. ${ }^{2}$ \\ ${ }^{1}$ METAS, Bern, SWITZERLAND, ${ }^{2}$ PTB, Braunschweig, GERMANY \\ nina.basic@metas.ch
}

DOI 10.25039/x46.2019.PO063

\begin{abstract}
Diffuse reflectance measurements are usually performed using a single photodetector. However graininess measurements require also spatial resolution, thus the measurements are performed using a radiometric camera attached to the integrating sphere in the measurement geometry $\mathrm{d}: 0^{\circ}$. When performing such measurements on glossy samples, an interesting phenomenon was observed; a dark area corresponding to the specular excluded area. METAS and PTB have performed a systematic study on a set of samples having different values of gloss, lightness, and colour, using two different measurement setups. It was found that the size of the dark area could be calculated using geometric relations of the measurement setup. Furthermore, the luminance factor of the dark area, compared to its surround can be correlated with the gloss value of the sample; the glossier the sample, the greater the difference between the dark area and its surround.
\end{abstract}

Keywords: Diffuse Reflectance, Gloss, Graininess

\section{Introduction}

Coatings with special effect pigments are becoming widely used in many industries, including automotive, cosmetics, textile, and others, due to their eye-catching colour and texture effects [Streitberger 2008, Faulkner 2009, Maile 2005, Wißling 2005]. Special effect pigments are embedded in a transparent binder, and oriented imperfectly parallel to the coatings surface. The visual effects of such a coating depend not only on the optical properties of a binder and special effect pigments, but also on the orientation, size, and concentration of special effect pigments. Imperfectly oriented pigments result in two visual texture effects, referred to as sparkle and graininess. The sparkle, also known as glint, is observed under directional illumination. The effect is seen similarly as stars on a night sky, with point light sources on a dark background. When directional illumination is changed to diffuse illumination, the graininess, also known as diffuse coarseness, can be observed [Kirchner 2007, Ferrero 2013, Ferrero 2018].

Since graininess is observed under diffuse illumination, an integrating sphere is used for its measurements. Moreover, since the spatial resolution of the sample is also required, a camera is used as a detector. The camera is placed at the port opposite to the sample port, avoiding any distortions of the image due to the perspective. The measurement geometry of such a setup is the strict specular excluded $\mathrm{d}: 0^{\circ}$ geometry. During graininess measurements of glossy samples, performed within the EMPIR project BiRD ("Bidirectional reflectance definitions"), an interesting phenomenon has been observed; a dark area corresponding to the specular excluded area. METAS and PTB have performed additional measurements, using two different measurement setups, in order to explain the phenomenon and relate it to measurement setup and sample parameters. PTB measured glossy coloured and neutral samples, varying the distance of the camera to the integrating sphere port. METAS on the other hand measured gloss scale (4 grey values, 7 values of glossiness per grey value) along with a mirror (as an example of a perfectly glossy material) while varying the integrating sphere port sizes.

The CIE recommended measurement geometries for diffuse reflectance [CIE 2018] allow the inclusion or exclusion of the specularly (regularly) reflected light by using either di: $8^{\circ}$, or de: $8^{\circ}$ measurement geometry. The difference between the two measurement geometries is, to a first approximation, an indication of glossiness [NPL 2006]. Following this idea, the samples 
presented in the paper have various gloss level. This allowed us to also explore the relation of the sample's gloss value to the difference in luminance factor between the dark and bright area, using only one measurement, instead of two $\left(\mathrm{di}: 8^{\circ}\right.$ and de: $\left.8^{\circ}\right)$.

The paper first presents a simple theoretical model for calculation of the diameter of the dark area. This is followed by the description of both measurements setups, as well the samples used. Afterwards, the predicted theoretical diameters are compared to the measured ones. The paper concludes with the relation of gloss level to the difference in luminance factor for the dark and the bright area.

\section{Theory}

Spatially resolved measurements of diffuse reflectance are performed with an integrating sphere and a camera as the detector. In order to avoid any distortions of the image, the camera is usually placed at the port directly opposite to the sample. The described setup corresponds to measurement geometry $\mathrm{d}: 0^{\circ}$. Even though it is not explicitly specified, the specular component is not included. This can be understood easier if one reverses the directions and takes a look at $0^{\circ}: \mathrm{d}$ measurement geometry. The specular component in this case follows the same beam path as the incident light. Because of reciprocity, the same applies also when measuring in geometry $\mathrm{d}: 0^{\circ}$.

When measuring glossy samples with a large specular component, a dark area was observed. Figure 1 shows a luminance factor image of a mirror measured in measurement geometry $\mathrm{d}: 0^{\circ}$, where a dark circular area can be clearly seen.

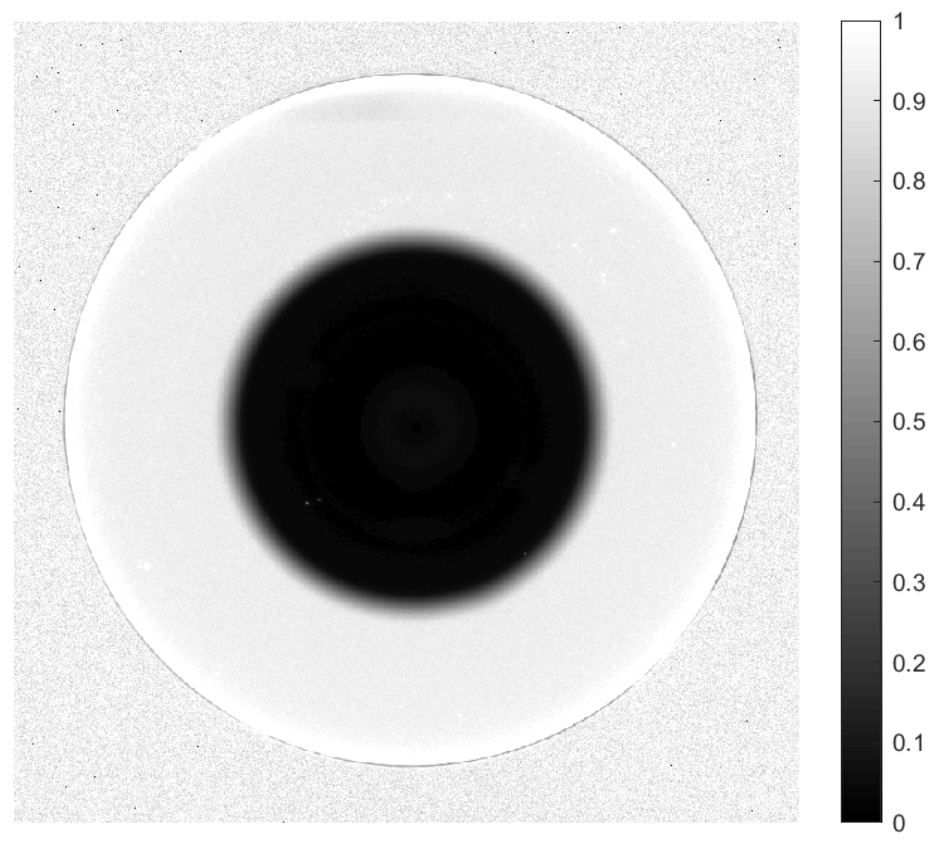

Figure 1 - Luminance factor image of a mirror measured in geometry $\mathrm{d}: 0^{\circ}$. The circular dark area, corresponding to the specular excluded component, can be clearly seen in the middle. The outer circle corresponds to the sample port, a circular opening with diameter of $30 \mathbf{m m}$.

The dark area is assumed to be an out of focus reflection of the camera port. Therefore, its size can be calculated from simple geometric relations. Figure 2 shows a schematic of such a measurement setup. The sample can be placed either at the middle of the integrating sphere, or at the side (at sample port). Depending on the sample placement, the light source is placed either behind the sample (sample in the middle of the integrating sphere), or at a light source port placed $90^{\circ}$ from the sample and camera ports (sample placed at sample port). In the latter case, special care must be taken so that the light does not directly illuminate neither the sample nor the camera's field of view (FOV). The camera is placed at the port opposite to the 
sample, either directly at the port or at a certain distance. It is focused on the sample. The relevant dimensions of the system are marked in the schematic (Figure 2).

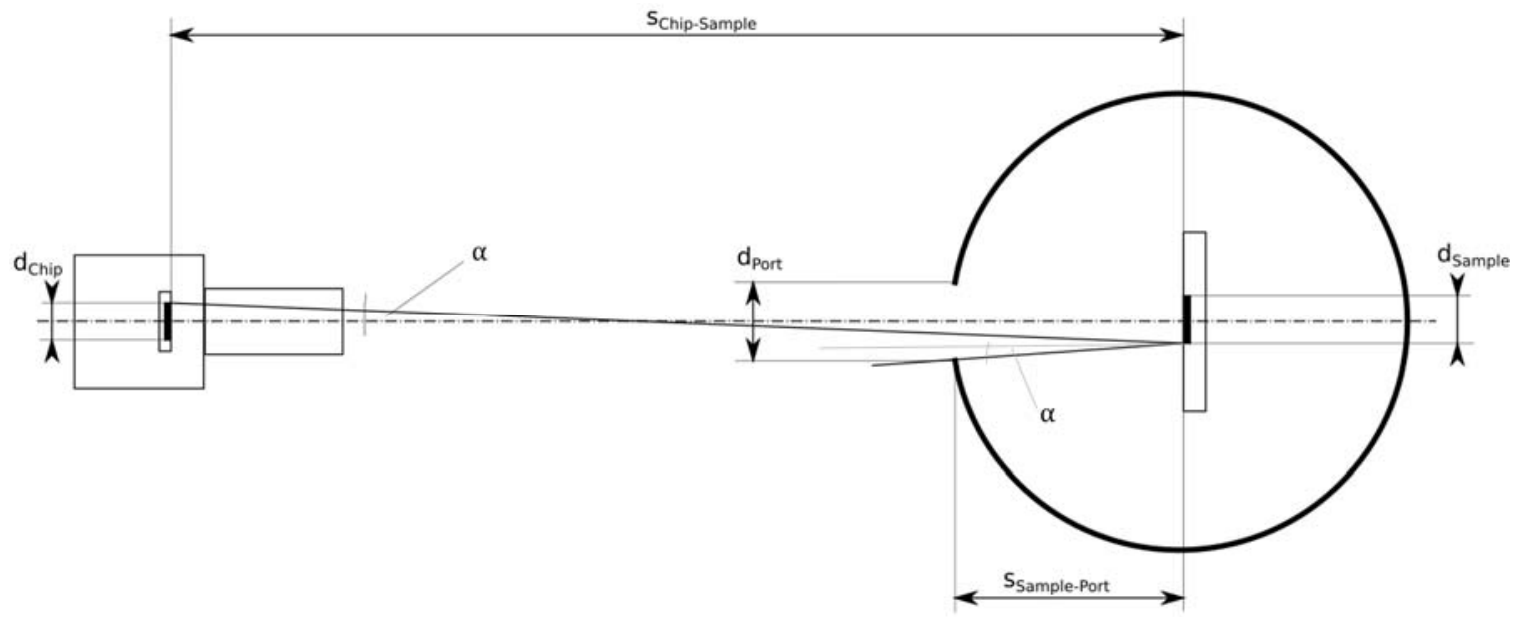

Figure 2 - Schematic of a measurement setup for spatially resolved diffuse measurements. The relevant parameters marked in the figure, are explained in the text.

The expression for the size of the dark area is derived from geometric relations for circular apertures for simplicity and practicality. First, the observation half angle of dark area, $\alpha$, is expressed in two ways:

$$
\begin{aligned}
& \frac{d_{\text {chip }}+d_{\text {sample }}}{2 s_{\text {chip-sample }}}=\tan \alpha \\
& \frac{d_{\text {port }}-d_{\text {sample }}}{2 s_{\text {sample-port }}} \cong \tan \alpha
\end{aligned}
$$

where $d_{\text {chip }}$ is the diameter of the dark area on camera's chip, $d_{\text {sample }}$ is the diameter of dark area on sample (as seen by the camera), $s_{\text {chip-sample }}$ is the distance between the chip and the sample, $d_{\text {port }}$ is the diameter of the camera port, and $s_{\text {sample-port }}$ is the distance between the sample and the camera port. It should be mentioned, that equation (1) only holds up for objectives that can be well approximated as a thin lens. If this is not the case, but the distance between the principal planes of the objective is known, the distance from the camera chip to the sample should be modified accordingly. The diameter of the dark area on camera's chip is expressed using the magnification factor of camera, $V$ :

$$
d_{\text {chip }}=d_{\text {sample }} V=d_{\text {sample }} \frac{s_{\text {pixel }}}{F O V_{\text {pixel }}}
$$

where $s_{\text {pixel }}$ is the physical edge length of camera's pixel, and $F O V_{\text {pixel }}$ is the edge length of a single pixel's FOV on the sample. Combining and rearranging equations (1)-(3), we are left with the expression for the diameter of the dark area:

$$
d_{\text {sample }}=\frac{d_{\text {port }} \cdot s_{\text {chip-sample }}}{s_{\text {sample-port }} \cdot(1+V)+s_{\text {chip-sample }}}
$$

\section{Experimental}

\subsection{PTB measurement setup}

The measurement setup at PTB (Figure 3 ) features an integrating sphere with a diameter of $500 \mathrm{~mm}$. The sample is positioned in the centre of the sphere, with the illumination source (a halogen lamp) behind the sample. A baffle between both ensures that neither the sample, nor the detector are illuminated directly. A $45 \mathrm{~mm}$ in diameter observation port allows measurements of sample surfaces in d:0 $0^{\circ}$ geometry. As the detector, a QSI 520i CCD camera with a Kodak KAI-2020M Chip was used. The CCD chip features square pixels with $7.4 \mu \mathrm{m}$ 
edge length. The used objective is a Sigma Mini-Wide II with a fixed focal length of $28 \mathrm{~mm}$. The detector is positioned on an optical rail, so that the distance between camera and sample can be adjusted.

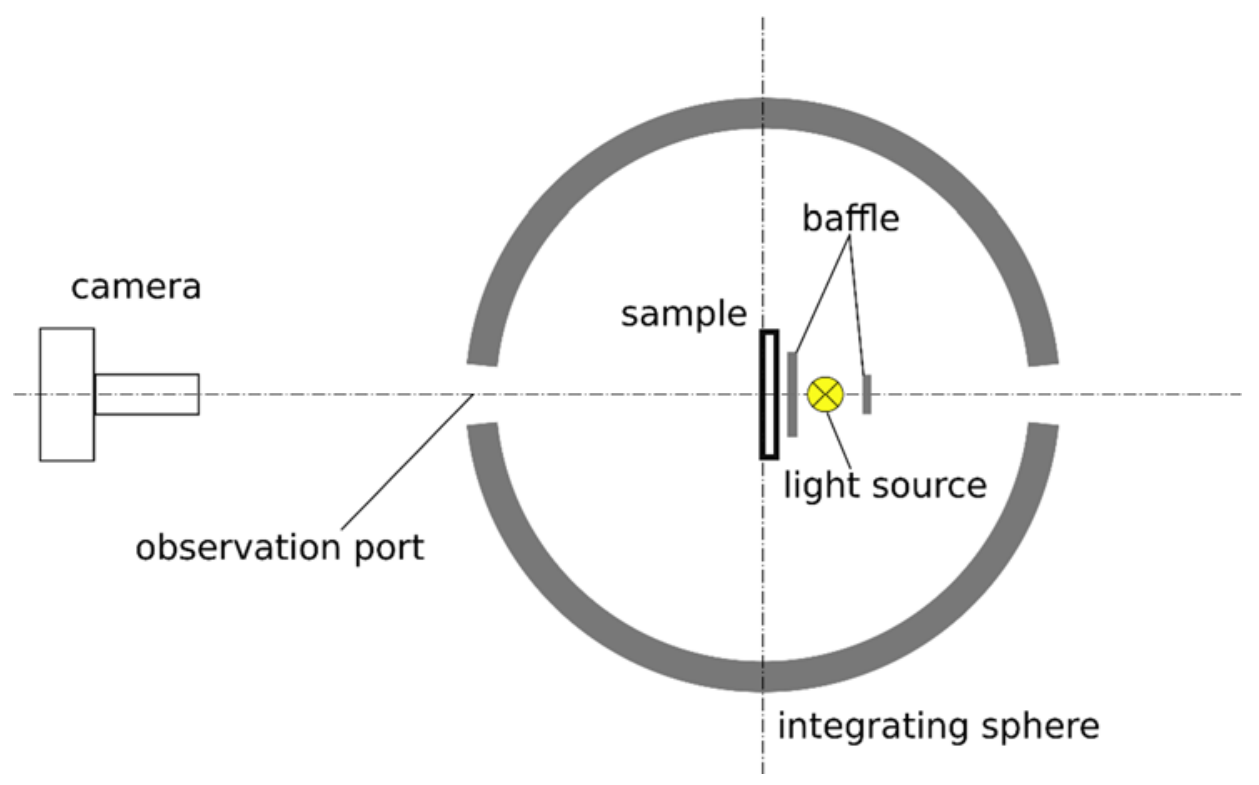

Figure 3 - Measurement setup at PTB.

\subsection{METAS measurement setup}

METAS measurement setup consists of an integrating sphere with $150 \mathrm{~mm}$ diameter with Barium sulphate coating. Five $30 \mathrm{~mm}$ diameter ports allow one to measure reflection in measurement geometry $\mathrm{d}: 0^{\circ}$ as well as in de: $8^{\circ}$ and di: $8^{\circ}$. The sphere is illuminated from the side with a spectrally tunable light source. The divergence of the source is small enough to not directly illuminate the sample or the camera's FOV. The detector is a CCD camera with a $1 / 1.8$ " sensor, placed directly at the camera's port. The pixels are squared with length size of $4.4 \mu \mathrm{m}$. The camera is used in combination with an objective having focal length $f=16 \mathrm{~mm}$ and an extension ring of $0.5 \mathrm{~mm}$. The objective's length is $28.2 \mathrm{~mm}$, and its back focal length is $13.1 \mathrm{~mm}$. The resolution of the detector system is $45 \mu \mathrm{m}$ per pixel.

All the ports of the integrating sphere, except the light source port, have a diameter of $30 \mathrm{~mm}$. Using port plugs and port reducers having the same optical properties as the integrating sphere itself, the diameters of the ports were being able to vary without changing the optical properties of the sphere. The light source port has a diameter of $7 \mathrm{~mm}$.

For measurement geometry $\mathrm{d}: 0^{\circ}$ (Figure 4 left), the camera was placed at the port opposite of the sample. The sample port was kept at the diameter of $30 \mathrm{~mm}$, while the camera port diameter was varied $\left(10,15\right.$, and $30 \mathrm{~mm}$ ). For measurement geometry $\mathrm{d}: 8^{\circ}$ (Figure 4 right), the camera was placed at a port having an angle of $8^{\circ}$ to the sample port's normal. The sample port size was again kept at $30 \mathrm{~mm}$, and the camera port was kept at $15 \mathrm{~mm}$. The port that influences the dark spot is the specular port, which is placed at $-8^{\circ}$ from the sample port's normal. For measurement geometry di: $8^{\circ}$, the specular port was kept closed, while for measurement geometry de: $8^{\circ}$, the port was left open, and its diameter was varied $(10,15$, and $30 \mathrm{~mm})$. 

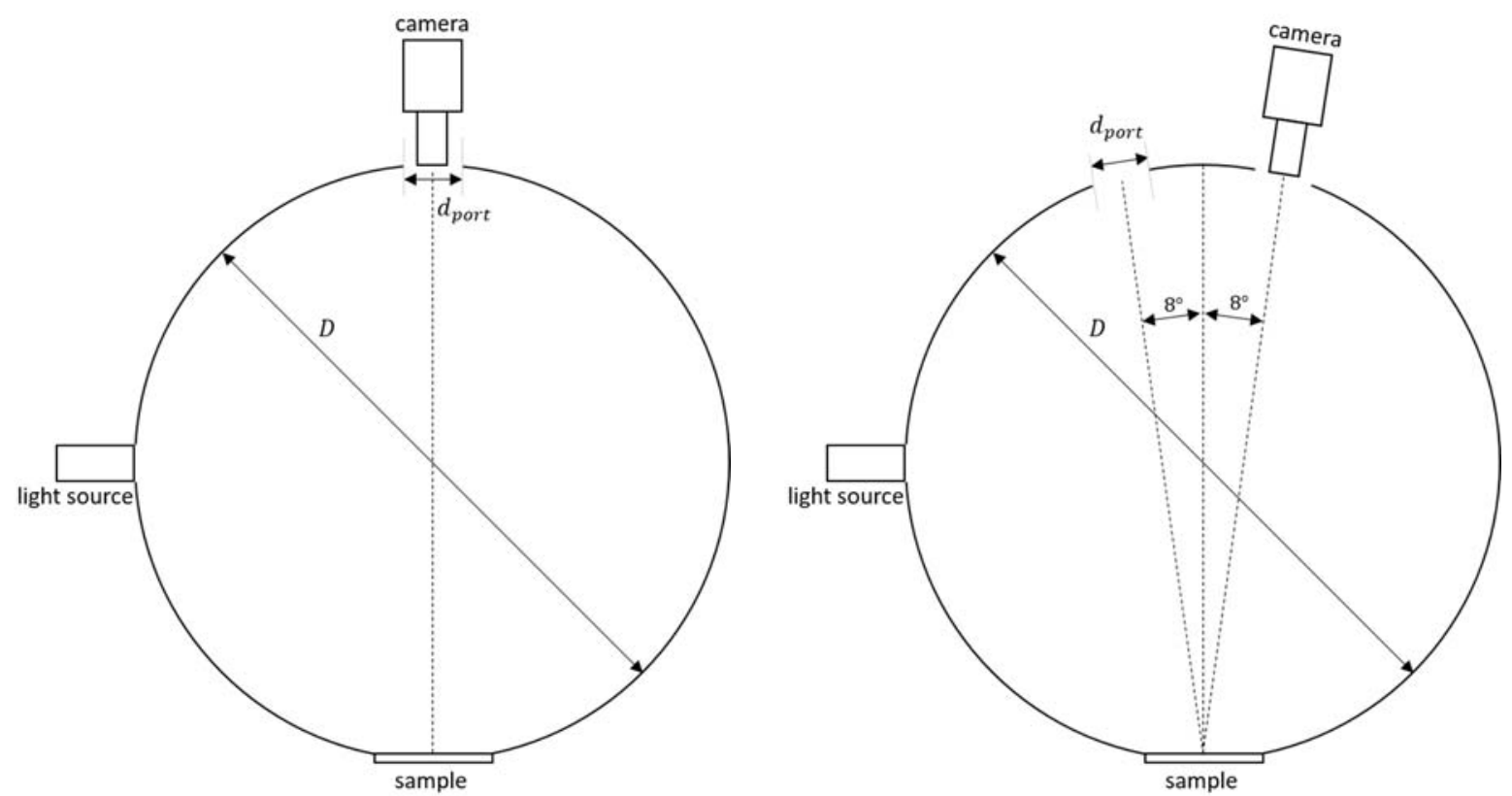

Figure 4 - Schematic of METAS measurement setup for measurement geometry d: $0^{\circ}$ (left) and measurement geometry $\mathrm{d}: 8^{\circ}$. The parameters of the setup are gathered in Table 1.

Table 1- Relevant parameters of PTB and METAS measurement setup.

\begin{tabular}{|c|c|c|c|c|}
\hline description & symbol & unit & METAS & PTB \\
\hline $\begin{array}{l}\text { diameter of integrating } \\
\text { sphere }\end{array}$ & $D$ & $\mathrm{~mm}$ & 150 & 500 \\
\hline diameter of camera port & $d_{\text {port }}$ & $\mathrm{mm}$ & $\begin{array}{l}10 / 15 / \\
30\end{array}$ & 45 \\
\hline $\begin{array}{l}\text { distance from chip to } \\
\text { sample }\end{array}$ & $S_{\text {chip-sample }}$ & $\mathrm{mm}$ & 191.8 & 664 / 784 / $902 / 1023$ \\
\hline $\begin{array}{l}\text { distance from sample to } \\
\text { camera port }\end{array}$ & $S_{\text {sample-port }}$ & $\mathrm{mm}$ & 150 & 250 \\
\hline $\begin{array}{l}\text { physical edge length of } \\
\text { camera's pixel }\end{array}$ & $S_{\text {pixel }}$ & $\mu \mathrm{m}$ & 4.4 & 7.4 \\
\hline $\begin{array}{l}\text { edge length of a single } \\
\text { pixel's FOV on the sample }\end{array}$ & $F O V_{\text {pixel }}$ & $\mu \mathrm{m}$ & 45 & $\begin{array}{l}\text { variable (dependent on } \\
\text { distance of camera to sample) }\end{array}$ \\
\hline
\end{tabular}

\subsection{Samples}

Two sets of samples were used. PTB used a total of six samples made from coated sheet metal. Three of the samples are achromatic black, grey and white and the other three samples are coloured red, blue and green. The samples gloss values are varying between 80 and $100 \mathrm{GU}$, save for the green sample, that is relatively mat with $35 \mathrm{GU}$. It should be mentioned that this kind of samples tends to show some form of surface curvature that might have additional effects on the results of the dark spot diameter. The flatness of the samples surfaces was not thoroughly investigated.

METAS used gloss scale and a mirror. The gloss scale consists of 4 different grey values; black, dark grey, light grey, and white. Each grey value has 7 different gloss values; gloss unit $2,6,12,30,50,75$, and 95 . The NCS gloss scale is referred to in the paper as 'grey value GUxx', where GUxx represents the gloss unit of the sample. The mirror was used as a sample having maximum gloss, since all the light is reflected in the specular direction. 


\section{Results and discussion}

Two sets of samples were measured using two different measurement setups. The measurement geometry and the measurement parameter were varied in order to explain the phenomena of the observed dark are on the luminance factor images.

A mirror was measured in measurement geometries $\mathrm{d}: 0^{\circ}$, and de: $8^{\circ}$ with varying port diameters: $10 \mathrm{~mm}, 15 \mathrm{~mm}$, and $30 \mathrm{~mm}$. The diameter of the dark area was determined using the $25 \%, 50 \%$, and $75 \%$ transition point between the dark and the bright average. The measured diameters are shown in Figure 5, along with the theoretical values determined with the Equation 4. From the figure, we can clearly see that the $50 \%$ transition point is the correct way to go.

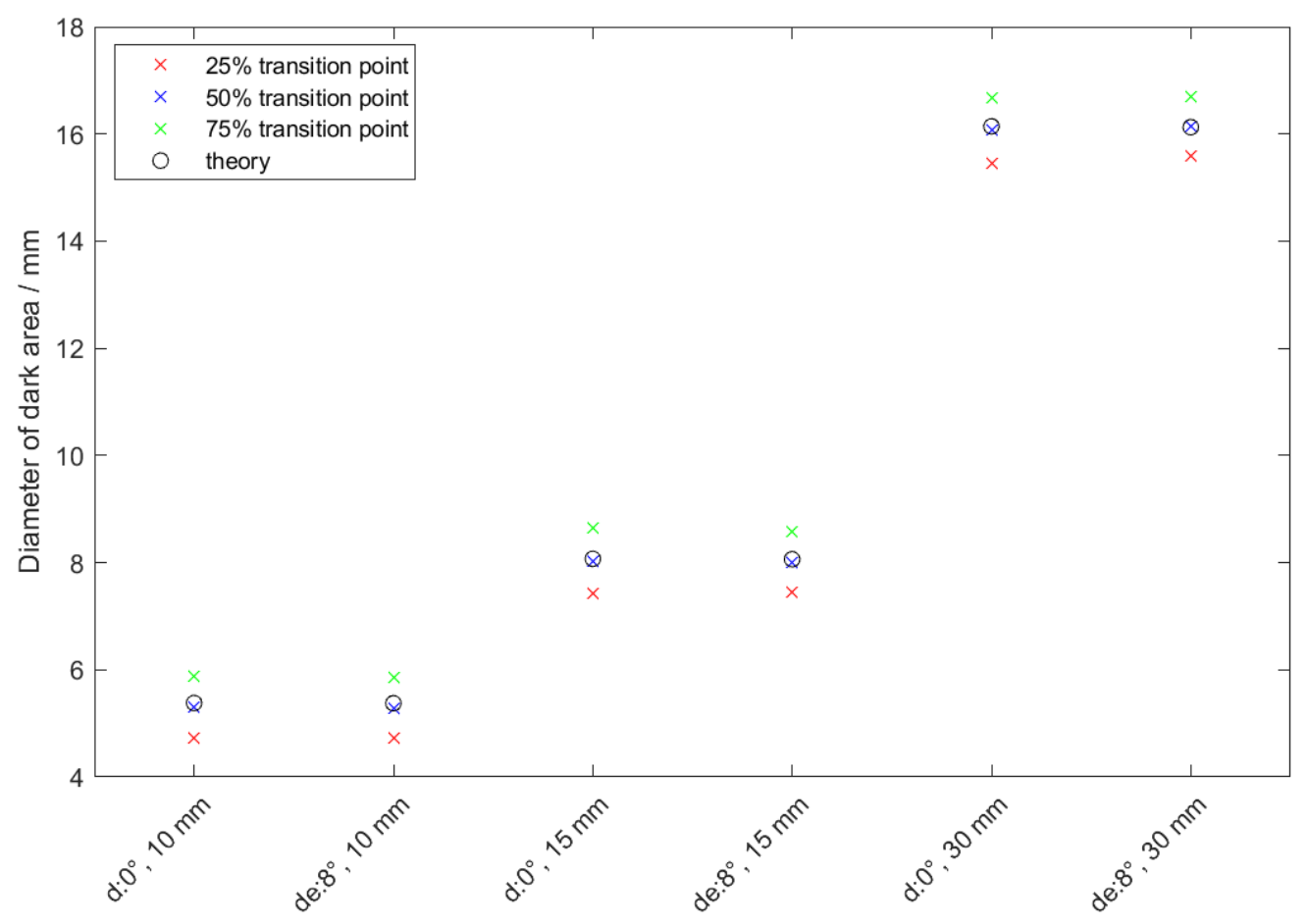

Figure 5 - Measured and calculated diameter of the dark area for mirror in measurement geometries d: $0^{\circ}$ and de: $8^{\circ}$. The measured diameter was estimated using $25 \%, 50 \%$, and $75 \%$ transition point between the dark and bright average.

For both measurement geometries, the measured diameter using $50 \%$ transition point, matches the theoretical diameter. Furthermore, the measurement geometry does not affect the results; as long as the parameters in Equation 4 are appropriately corrected for (the pixel's FOV has to be multiplied by $\cos 8^{\circ}$ to account for the camera's perspective). The diameter using $25 \%$ transition point underestimates the diameter while the $75 \%$ transition point overestimates it. Therefore, all the following measured diameters presented in the paper are determined using $50 \%$ transition point. The difference in diameters determined from different transition points shows that the dark area does not have a clear edge even for a highly glossy sample, such as a mirror. This follows from the fact that the camera focuses on the sample, and the dark area is an out of focus reflection of the camera port.

The edge of the dark area gets more blurry when the sample gets less glossy. Figure 6 left shows a luminance factor image of black GU95 sample measured in de: $8^{\circ}$ with camera port diameter of $30 \mathrm{~mm}$. In comparison with Figure 1, which shows the mirror measured in the geometry $\mathrm{d}: 0^{\circ}$, the blurring of the dark area edge becomes apparent. When samples are measured in geometry di: $8^{\circ}$, the specular component is included, and the dark area disappears. Figure 6 right shows black GU95 measured in geometry di: $8^{\circ}$. Instead of the dark area, only a thin circle is observed. The circle is a consequence of a not perfect integrating 
sphere. The port reducer used to close the specular port does not cover the port perfectly and thus the thin circle can be observed.
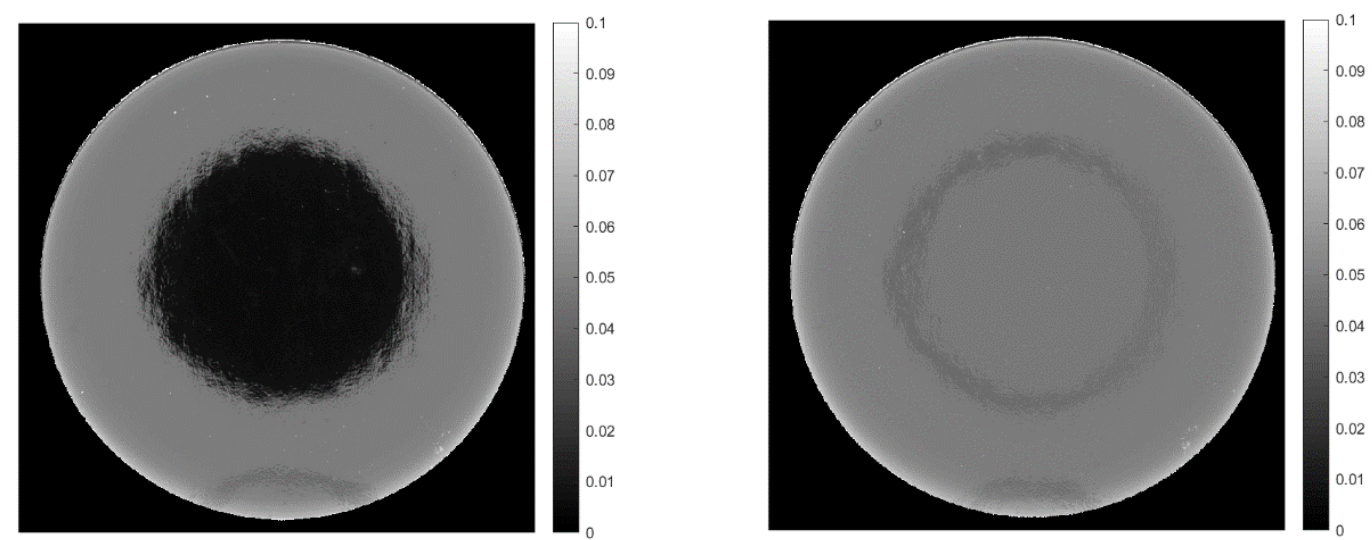

Figure 6 - Luminance factor images of black GU95 in measurement geometry de: $8^{\circ}$ with camera port diameter of $30 \mathrm{~mm}$ (left), and in measurement geometry di: $8^{\circ}$ (right).

Measurement with varying sample port diameter for all measurement geometries, and varying camera port diameter for geometries de: $8^{\circ}$, and di: $8^{\circ}$ were also performed. However, the size of these diameters does not affect the size of the dark area, as long as the sample port diameter is larger than the observable dark area.

Table 2 gathers the calculated and measured diameters of the dark spot for measurements of dark grey gloss scale in measurement geometry $\mathrm{d}: 0^{\circ}$, with varying camera port diameter. The calculated diameter holds well for high gloss samples. When the gloss value decreases, the diameter of the dark area increases. Matt samples, having the two lowest gloss levels (GU 2, and GU 6), have a measured diameter that spans almost the entire sample port, which has a diameter of $30 \mathrm{~mm}$. The measured diameter is taken using the $50 \%$ transition point between the dark and the bright average. If one would take a higher transition point, the measured diameter would match the sample port diameter.

Table 2 - Calculated and measured diameters of the dark spot for dark grey gloss scale samples measured in geometry $\mathrm{d}: 0^{\circ}$.

\begin{tabular}{|c|c|c|c|c|}
\hline & $\begin{array}{l}\text { port diameter / } \\
\mathrm{mm}\end{array}$ & 30 & 15 & 10 \\
\hline & theoretical / mm & 16.14 & 8.07 & 5.38 \\
\hline \multirow{7}{*}{ 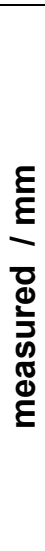 } & GU 95 & 15.68 & 7.90 & 5.24 \\
\hline & GU 75 & 15.38 & 8.03 & 5.40 \\
\hline & GU 50 & 15.90 & 9.36 & 8.01 \\
\hline & GU 30 & 16.77 & 13.03 & 14.47 \\
\hline & GU 12 & 20.23 & 18.59 & 19.76 \\
\hline & GU 6 & 26.01 & 25.74 & 27.09 \\
\hline & GU 2 & 27.50 & 26.42 & 28.44 \\
\hline
\end{tabular}


Figure 7 shows the difference between measured and calculated diameter of the dark area for all samples measured in measurement geometry $\mathrm{d}: 0^{\circ}$, with varying either the port diameter (METAS setup) or varying the distance from camera to sample (PTB setup). The samples were separated into three categories based on level of gloss; high gloss with gloss units of 70 and higher, medium gloss with gloss units of 10 to 70 , and low gloss, with gloss units below 10. For each category, the mean difference is plotted in the figure, along with standard deviation as error bars. The figure confirms the previous statement; the difference between measured and calculated diameter increases with decreasing gloss value.

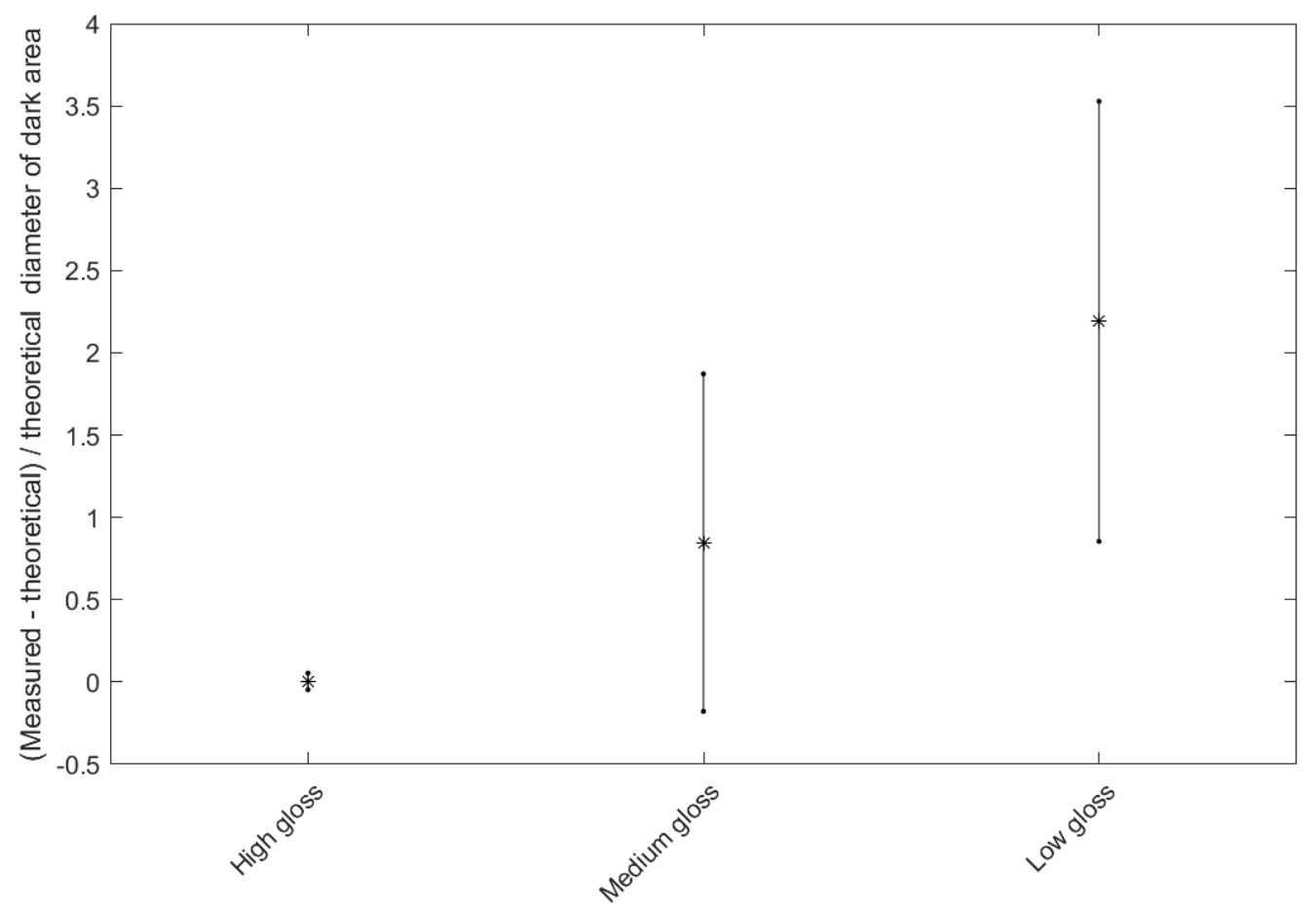

Figure 7 - Relative mean difference between calculated and measured diameter of dark area. Samples were measured in measurement geometry $\mathrm{d}: 0^{\circ}$, and separated into three categories; high gloss (gloss unit > 70), medium gloss $(10>$ gloss unit $>70$ ), and low gloss (gloss unit < 10). The error bars are standard deviation for each category.

The difference between the measurement of diffuse reflectance with specular component excluded or included gives an indication of glossiness. The dark area in our measurements represents the specular excluded part, while the bright area corresponds to the specular included part. Therefore, the difference between the two values should provide an estimation of the glossiness level. Figure 8 plots this difference against the gloss unit for gloss scale samples with gloss unit of 12 or higher. Samples with lower gloss unit were excluded since the measured diameter spans almost whole sample port, meaning that the dark area is no more observable. Measurement parameters were kept constant; geometry $\mathrm{d}: 0^{\circ}$ with camera port diameter of $15 \mathrm{~mm}$. 


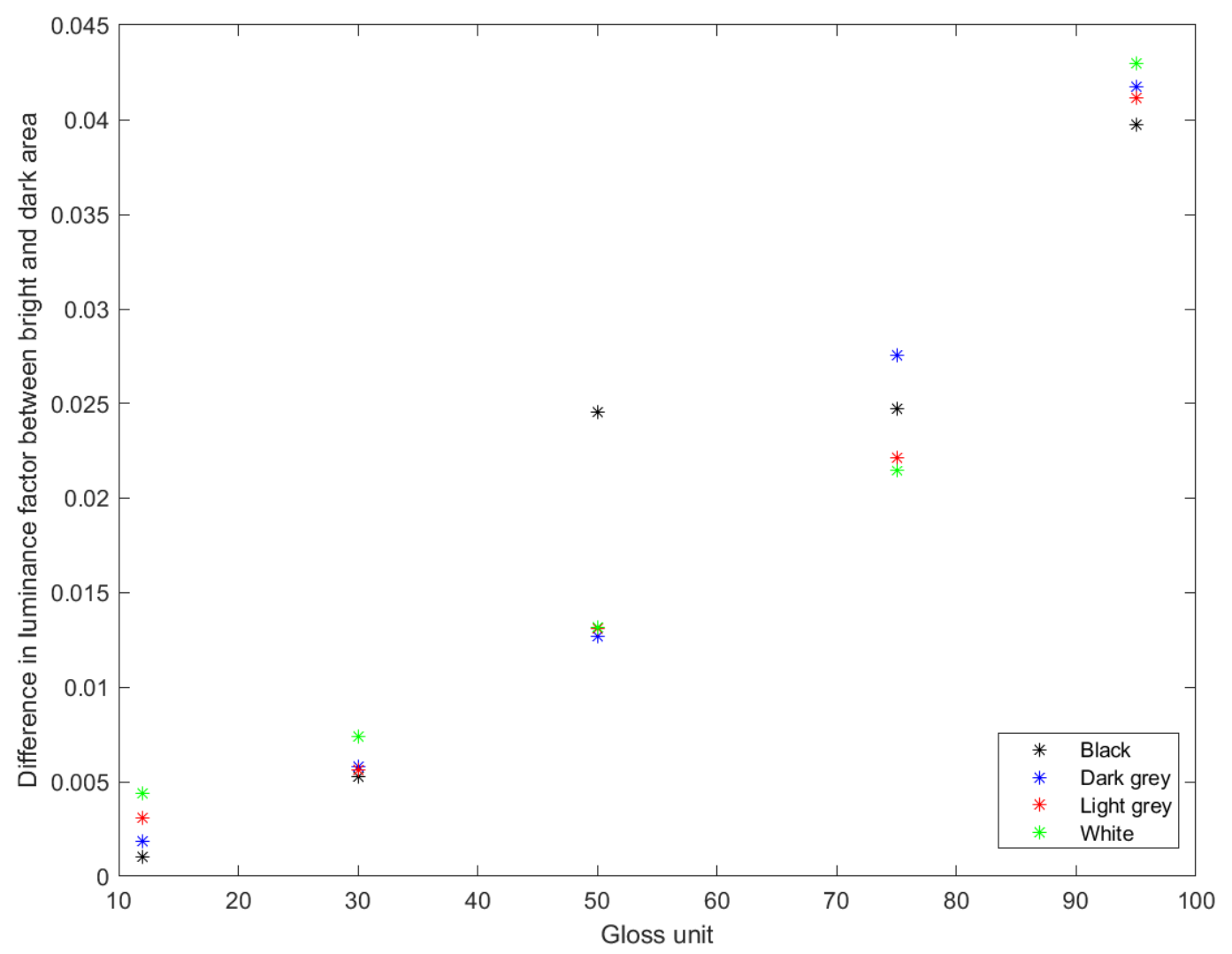

Figure 8 - Difference in luminance factor between bright and dark average against gloss unit for gloss scale samples measured in geometry d:0 $0^{\circ}$ with camera port diameter of $15 \mathrm{~mm}$.

\section{Conclusions}

METAS and PTB have performed spatially resolved diffuse reflectance measurements on a set of samples having different values of gloss, lightness, and colour. When using specular excluded measurement geometry, a dark area was observed. The size of the dark area was correlated with the measurement geometry setup (size of integrating sphere port, distance from the sample to the camera) and the results fit well for samples with high gloss. When the glossiness of the sample decreases, the diameter as well as the luminance factor of the dark area increases. For a very matte sample, the dark area covers the whole sample, while its luminance factor is the same as for the specularly included geometry.

Furthermore, the difference in luminance factor between the dark and bright area was correlated with the gloss value of the sample. The indication of glossiness so far required sample to be measured twice; once in di: $8^{\circ}$ geometry, and in once in de: $8^{\circ}$ geometry. Our method only requires one measurement; either in $\mathrm{d}: 0^{\circ}$, or de: $8^{\circ}$ geometry. It could be a fast way to approximate the glossiness level of the sample.

\section{References}

CIE 2018. CIE 15:2018. Colorimetry, 4th Edition. Vienna: CIE.

FAULKNER, E. B., and SCHWARTZ, R. J. 2009. High Performance Pigments, 2nd Ed. Weinheim: Wiley-Vch.

FERRERO, A., CAMPOS, J., RABAL, A. M., and PONS, A., 2013. A single analytical model for sparkle and graininess patterns in texture of effect coatings. Opt. Express 21, 2681226819. 
FERRERo, A., VELÁZquez, J. L., PERALES, E., CAMPOS, J., and MARTíNEZ VERdú, F. M. 2018. Definition of a measurement scale of graininess from reflectance and visual measurements. Opt. Express 26, 30116-30127.

KIRCHNER, E., van den KIEBOOM, G. J., NJO, L., SUPÈR, R., and GOTTENBOS, R. 2007. Observation of visual texture of metallic and pearlescent materials. Col. Res. Appl. 32, 256-266.

MAILE, F. J., PFAFF, G., and REYNDERS, P. 2005. Effect pigments-past, present and future. Prog. organic coatings 54, 150-163.

NPL 2006. Good practice guide for the measurement of gloss.

STREITBERGER, H.-J., and DOSSEL, K.-F. 2008. Automotive Paints and Coatings, 2nd Ed. Weinheim: Wiley-Vch.

WIßLING, P. 2006. Metallic Effect Pigments: Fundamentals and Applications. Hannover: Vincentz Network GmbH \& Co KG. 